\title{
A Study of Fair Bandwidth Sharing with AIMD-Based Multipath Congestion Control
}

\author{
Dizhi Zhou, Wei Song, Member, IEEE, and Yu Cheng, Senior Member, IEEE
}

\begin{abstract}
The multi-homed capability offers a good opportunity to explore multipath transmission. To ensure fair bandwidth sharing between multipath flows and legacy single-path flows, congestion control is an essential mechanism at the transport layer to regulate traffic flows for bandwidth consumption. However, in a multipath scenario, the congestion control algorithm of the dominant transport control protocol (TCP) may result in too aggressive behavior. In this paper, we consider the generic congestion control principle of additive increase and multiplicative decrease (AIMD) and investigate two AIMD-based multipath congestion control solutions. We derive the conditions for AIMD parameters to ensure TCP-friendliness and satisfy two constraints for fair bandwidth sharing between multipath and single-path flows. Simulation results demonstrate that the AIMD-based multipath solutions well achieve the fairness goals.
\end{abstract}

Index Terms-Congestion control, multipath transmission, MPTCP, AIMD, additive increase multiple decrease.

\section{INTRODUCTION AND RELATED WORK}

A $\mathrm{N}$ increasing number of network devices nowadays are equipped with multiple interfaces, such as multi-radio mobile devices and multi-homed Internet servers. The multihomed capability of these devices offers a good opportunity to explore multipath transmission so as to optimize the quality of service (QoS) of bandwidth-intensive applications such as video streaming and conferencing [1]. Although the transport control protocol (TCP) is the de facto standard for Internet applications, TCP is subject to some inherent limitations that restrict its direct deployment to multipath transmission. For example, the TCP congestion control algorithm follows the increase-by-one and decrease-by-half principle, which often introduces a highly fluctuating throughput that is not well suited for QoS assurance to real-time applications [2].

Although many non-TCP transport protocols are proposed, in view of the dominant role of TCP, it is important to ensure TCP-friendliness and avoid unfair bandwidth sharing between TCP flows and non-TCP flows. In [2], Cai et. al. analyze a single-path TCP-friendly protocol (known as DAIMD) based on the additive-increase and multiplicative-decrease (AIMD) principle for multimedia applications. In AIMD, the source additively increases the congestion window by $\alpha$ for each round trip time (RTT) and multiplicatively decreases the window size to $\beta$ of its previous value whenever there is a congestion indication (e.g., duplicate acknowledgements). Actually, TCP is a special case of AIMD with $\alpha=1$ and

Manuscript received December 10, 2012. The associate editor coordinating the review of this letter and approving it for publication was J.-C. Chen.

This research was supported by a research grant from the Natural Sciences and Engineering Research Council (NSERC) of Canada.

D. Zhou and W. Song are with University of New Brunswick, Fredericton, Canada (e-mail: \{q5frc, wsong\}@unb.ca).

Y. Cheng is with Illinois Institute of Technology, Chicago, USA (e-mail: cheng@iit.edu).

Digital Object Identifier 10.1109/WCL.2013.022213.120904 $\beta=0.5$. It is found in [2] that an AIMD flow and a TCP flow obtain a fair share if the $(\alpha, \beta)$ of the AIMD flow satisfies

$$
\alpha=\frac{3(1-\beta)}{1+\beta}
$$

where $0<\alpha<3$ and $0<\beta<1$. Two AIMD flows with different $(\alpha, \beta)$ pairs share the bandwidth fairly if

$$
\frac{\alpha_{1}}{\alpha_{2}}=\frac{\left(1+\beta_{2}\right)\left(1-\beta_{1}\right)}{\left(1-\beta_{2}\right)\left(1+\beta_{1}\right)}
$$

where $\alpha_{1}, \alpha_{2}>0$ and $0<\beta_{1}, \beta_{2}<1$.

In a multipath scenario, if the AIMD or TCP congestion control algorithm were applied independently over each individual path, a multipth flow would acquire a throughput much larger that of a single-path AIMD or TCP flow. Obviously, this aggressive behavior jeopardizes fair resource sharing. A multipath transport control protocol (MPTCP) [3] is recently standardized to extend TCP with the multipath capability by binding TCP subflows over multiple paths. Particularly, a coupled congestion control algorithm [4] is designed to ensure that a multipath flow should a) perform at least as well as a single-path flow would on the best of the paths available to it; and b) take no more capacity than a single-path flow would obtain when experiencing the same loss rate [5]. The fairness requirement in b) basically guarantees that an MPTCP flow does no harm to regular single-path TCP flows. The MPTCP congestion control algorithm works as follows:

- Once the source receives an acknowledgement (ACK) from path $r$, it increases the congestion window of path $r$ by $\min \left(a / w_{\text {total }}, 1 / w_{r}\right)$; and

- Once the source receives a congestion signal from path $r$, it decreases the congestion window $w_{r}$ of path $r$ by $w_{r} / 2$.

Here, $w_{r}$ is the current congestion window size of TCP subflow on path $r$, and $w_{\text {total }}$ is the total congestion window size of all TCP subflows. The aggressiveness factor $a$ is defined to respect the above throughput constraints, given by

$$
a=w_{\text {total }} \frac{\max _{r} w_{r} / R T T_{r}^{2}}{\left(\sum_{r} w_{r} / R T T_{r}\right)^{2}}
$$

where $R T T_{r}$ is the RTT of path $r$, while $\min \left(a / w_{\text {total }}, 1 / w_{r}\right)$ guarantees that each MPTCP subflow does not increase its congestion window faster than a single-path TCP flow with the same window size and does no harm to single-path TCP flows on the same path.

Although there were many studies on multipath routing with TCP [6], this paper focuses on the friendliness and fairness of multipath flows with single-path TCP and AIMD flows, which is not well explored in the literature [7]. Specifically, we investigate multipath congestion control based on the more generic AIMD principle to support a wide range of applications with diverse QoS requirements. To achieve fair bandwidth sharing among single-path AIMD flows and multipath AIMD flows, 
we derive some guidelines to select the congestion control parameters. Then, the resource sharing between single-path TCP flows and multipath MPTCP flows is viewed as a special case of the above general problem. Specifically, we aim to determine the AIMD parameters $(\alpha, \beta)$ so that

- Constraint 1 (C1): each of multipath and single-path flows obtains an even share of the overall bandwidths of all available paths; or

- Constraint 2 (C2): the aggregate throughput of a multipath flow should be the same if it were a single-path flow over the best path experiencing the same packet loss rate.

From a network operator's perspective, it is important to find a solution subject to the constraint $\mathrm{C} 1$ if non-differentiated service is provided and a single-path or multipath flow is charged the same. On the other hand, from an end user's perspective, multipath transmission can be promoted if a higher throughput is offered with no harm to single-path flows. It is worth mentioning that $\mathrm{C} 2$ does not imply that a multipath flow cannot achieve a throughput higher than that of a single-path flow on the best path. The constraint C2 is defined under the precondition of the same loss rate. A multipath flow is of course allowed to take the total bandwidth of all available paths if there is no competing traffic since a hypothetical single-path flow subject to no loss can get a very high throughput [5].

In the reminder of this paper, we analyze two AIMD-based multipath approaches in Section II and present experiment results in Section III and conclusions in Section IV.

\section{Aimd-BAsed Congestion Control Solutions FOR MUlTipATH TRANSMISSION}

In this section, we analyze two multipath congestion control solutions that are extended from DAIMD in a singlepath scenario [2] and the coupled algorithm in MPTCP [4], respectively. The analysis is focused on the AIMD parameters $(\alpha, \beta)$ that satisfy the above fairness constraints $\mathrm{C} 1$ and $\mathrm{C} 2$.

\section{A. Multipath Dynamic AIMD (MP-DAIMD)}

Consider a scenario in Fig. 1 with $M$ available paths. Assume that there are $N_{r}$ single-path AIMD flows on a path $r$. For any two single-path AIMD flows on the same path, their $(\alpha, \beta)$ pairs are selected to satisfy Eq. (2) so as to ensure fair bandwidth sharing. In addition, there is one multipath AIMD flow consisting of one subflow on each of the $M$ paths and the subflow on path $r$ takes the AIMD parameters $\left(\alpha_{r}, \beta_{r}\right)$. Here, $\left(\alpha_{r}, \beta_{r}\right)$ should be properly determined to ensure that the multipath flow obtains an even share of the total capacity as single-path AIMD flows. Denoting the bandwidth of path $r$ by $B_{r}$, we have the even share for each flow

$$
\lambda_{r}=\frac{\sum_{r} B_{r}}{\sum_{r} N_{r}+1} .
$$

When $N_{r} \lambda_{r}<B_{r}$, we have the ratio of the throughput occupied by each single-path flow over that of the multipath subflow on path $r$, given by

$$
\rho_{r}=\frac{\lambda_{r}}{B_{r}-N_{r} \lambda_{r}} .
$$

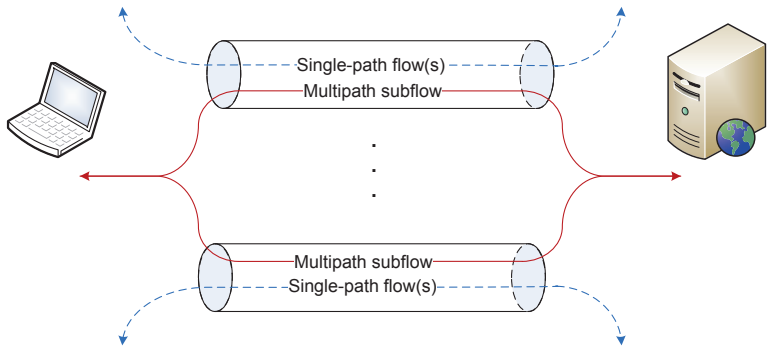

Fig. 1. Network topology.

If $N_{r} \lambda_{r} \geq B_{r}$, it implies that all flows cannot obtain an even share of the total bandwidth. To meet the constraint $\mathrm{C} 1$, we can select the paths occupied by the multipath flow by excluding the path of the smallest bandwidth and recalculating $\lambda_{r}$ as in (4) until all remaining paths satisfy $N_{r} \lambda_{r}<B_{r}$.

As analyzed in [2], the mean throughput of a flow in a steady state is proportional to its average congestion window size. Let $\bar{W}_{a, r}$ and $\bar{W}_{m, r}$ denote the average congestion window size of the single-path AIMD flows and that of the multipath subflow on path $r$, respectively. To satisfy $\mathrm{C} 1$, the sufficient and necessary condition is that

$$
\bar{W}_{a, r}=\rho_{r} \bar{W}_{m, r} .
$$

Following the analytical approach in [2] for single-path TCP and AIMD flows, we obtain

$$
\begin{aligned}
& \bar{W}_{a, r}=\frac{1+\beta}{2} \cdot \frac{C_{r} \alpha\left(\beta_{r}-1\right)}{\tau} \\
& \bar{W}_{m, r}=\frac{1+\beta_{r}}{2} \cdot \frac{C_{r} \alpha_{r}(\beta-1)}{\tau}
\end{aligned}
$$

where $C_{r}$ is the maximum capacity (in packets per RTT) of path $r$ and $\tau=\beta \alpha_{r}+\alpha \beta_{r}-\alpha-\alpha_{r}$. Then we have the following relation among the AIMD parameters of the singlepath flows and multipath subflows to meet the constraint $\mathrm{C} 1$ :

$$
\alpha_{r}=\frac{\alpha(\beta+1)\left(\beta_{r}-1\right)}{\rho_{r}(\beta-1)\left(\beta_{r}+1\right)} .
$$

Since the AIMD parameters of all single-path flows and multipath subflows should satisfy Eq. (1) to ensure fair bandwidth sharing, Eq. (9) can then be simplified to

$$
\alpha_{r}=\frac{3\left(1-\beta_{r}\right)}{\rho_{r}\left(1+\beta_{r}\right)} .
$$

Therefore, a multipath flow can select the AIMD parameters for two subflows on path $r$ and path $s$, respectively, so that

$$
\frac{\alpha_{r}}{\alpha_{s}}=\frac{\rho_{s}}{\rho_{r}} \frac{\left(1+\beta_{s}\right)\left(1-\beta_{r}\right)}{\left(1-\beta_{s}\right)\left(1+\beta_{r}\right)} .
$$

\section{B. Coupled Multipath AIMD (CMP-AIMD)}

MPTCP extends TCP with multipath capability and designs a coupled congestion control algorithm to meet the fairness constraint $\mathrm{C} 2$. To allow for a wide range of QoS provisioning, we can further apply the more generic AIMD principle and extends the coupled algorithm as follows:

- Once the source receives an ACK message from path $r$, it increases its congestion window $w_{r}$ for path $r$ by $\min \left(a_{r}^{\prime} / w_{m, \text { total }}, \alpha_{r} / w_{m, r}\right)$; and 
- Once the source receives a congestion signal from path $r$, it decreases its congestion window for path $r$ by $\beta_{r} w_{m, r}$. Here, $a_{r}^{\prime}$ is an aggressiveness factor for path $r,\left(\alpha_{r}, \beta_{r}\right)$ are the AIMD parameters for the subflow on path $r, w_{m, r}$ is the congestion window of the subflow on path $r$, and $w_{m, t o t a l}$ is the total congestion window size of the multipath subflows.

According to the constraint $\mathrm{C} 2$, a multipath flow takes no more capacity than any single-path TCP-friendly AIMD flow. The throughput of the multipath flow when subject to the same loss rate is upper bounded by that of the single-path flows, i.e.,

$$
\sum_{r} \frac{w_{m, r}}{R T T_{r}}=\max _{r} \frac{w_{a, r}}{R T T_{r}}
$$

where $w_{a, r}$ is the congestion window size of single-path flows on path $r$. The left side of (12) gives the aggregate throughput of the multipath flow, while the right side is the maximum throughput of single-path flows. For stability consideration, the increasing amount of the congestion window should be balanced with the decreasing amount in equilibrium. Hence,

$$
\left(1-p_{r}\right) \min \left(\frac{a_{r}^{\prime}}{w_{m, \text { total }}}, \frac{\alpha_{r}}{w_{m, r}}\right)=p_{r} w_{m, r} \beta_{r}
$$

where $p_{r}$ is the packet loss rate of path $r$. Likewise, the singlepath AIMD flows are subject to the same path loss and satisfy

$$
\left(1-p_{r}\right) \frac{\alpha_{r}}{w_{a, r}}=p_{r} w_{a, r} \beta_{r}
$$

Rearranging (14), we have

$$
\frac{p_{r}}{1-p_{r}}=\frac{1}{\left(w_{a, r}\right)^{2}} \cdot \frac{\alpha_{r}}{\beta_{r}} .
$$

The aggressiveness factor $a_{r}^{\prime}$ can then be determined to guarantee $\frac{a_{r}^{\prime}}{w_{m, \text { total }}} \leq \frac{\alpha_{r}}{w_{m, r}}$, by applying (15) to (13):

$$
a_{r}^{\prime}=\alpha_{r} w_{m, t o t a l} \frac{w_{m, r}}{\left(w_{a, r}\right)^{2}} .
$$

Considering the maximum throughput of single-path flows in (12), $a_{r}^{\prime}$ can be expressed in a form similar to (3) as follows:

$$
a_{r}^{\prime}=\alpha_{r} w_{m, \text { total }} \frac{\max _{s} w_{m, s} / R T T_{s}^{2}}{\left(\sum_{s} w_{m, s} / R T T_{s}\right)^{2}} .
$$

In a wireless environment, packet loss can be induced by congestion as well as link errors. On one hand, it is expected that the lower physical and link layers can address well packet erasure due to link errors. On the other hand, the above AIMD-based algorithm can be properly integrated with a wireless TCP solution as a sublayer below the multipath congestion control module. For example, in [8], a source node differentiates loss due to congestion or link errors based on explicit congestion notification (ECN). In the latter case, the source retransmits packets without decreasing the congestion window. Only if explicit congestion signal is received over a path should the source decrease its congestion window according to the AIMD parameters calculated above to ensure fairness. Therefore, in this case, the packet loss rate $p_{r}$ in (13) refers in particular to loss due to congestion.
TABLE I

SYSTEM PARAMETERS FOR EXPERIMENTS.

\begin{tabular}{l|c}
\hline \multicolumn{1}{c|}{ Parameter } & Value \\
\hline \hline Both path bandwidth in Scenario I & $10 \mathrm{Mbit} / \mathrm{s}$ \\
\hline Path 1 bandwidth in Scenario II & $4.27 \mathrm{Mbit} / \mathrm{s}$ \\
\hline Path 2 bandwidth in Scenario II & $7.73 \mathrm{Mbit} / \mathrm{s}$ \\
\hline Packet size & 500 bytes \\
\hline RED maximum queue length & 100 packets \\
\hline RED threshold & 60 packets \\
\hline
\end{tabular}

\section{EXPERIMENT RESULTS AND DISCUSSIONS}

We implement the MP-DAIMD and CMP-AIMD congestion control algorithms in $\mathrm{ns}-2$ by extending the existing TCP module. Simulations are conducted with the system parameters in Table I. Two scenarios are considered for the topology in Fig. 1, where two paths have the same bandwidth (Scenario I) or different bandwidths (Scenario II). There is one multipath subflow on each path as well as one or two AIMD singlepath flows (one on path 1 and two on path 2). Random early detection (RED) scheduling is used to ensure that every flow or subflow on the same path has the same opportunity to receive a congestion signal such as triple duplicate acknowledgements. In addition, we use saturated traffic model at the source and a constant packet size. This is because varying traffic may cause side effects that hinder proper interpretation of results.

Fig. 2(a) shows the throughput of three single-path AIMD flows and the total throughput of a multipath MP-DAIMD flow in Scenario I. The AIMD parameters $\left(\alpha_{r}, \beta_{r}\right)$ for each subflow can be derived by Eqs. (10) and (11). Two examples are illustrated in Fig. 2(a). In each case, we select two pairs of AIMD parameters $\left(\alpha_{r}, \beta_{r}\right)$ for the two subflows of the MPDAIMD flow and show them as the labels for the $\mathrm{x}$-axis. Here, we use the same $\beta_{r}$ for the two subflows over the two paths. As seen in Fig. 2(a), the multipath MP-DAIMD flow evenly shares the bandwidth with the other three single-path AIMD flows, which meets the design constraint C1. Further, Fig. 2(b) shows the corresponding throughput of single-path AIMD flows and the multipath MP-DAIMD flow in Scenario II, where the bandwidth of path 2 is 1.8 times that of path 1 . It is clearly observed in Fig. 2(b) that MP-DAIMD also achieves fair sharing with respect to $\mathrm{C} 1$ in the heterogeneous case.

From the end user's perspective, the fairness constraint $\mathrm{C} 2$ is defined to ensure that a multipath flow does not take more bandwidth than the single-path flows when subject to the same loss rate. The extended congestion control solution CMP-AIMD aims to meet the constraint C2. While the AIMD parameters are selected to satisfy Eq. (1), the aggressiveness factor $a_{r}^{\prime}$ is dynamically adapted according to (17). Given $\left(\alpha_{r}, \beta_{r}\right)=(1.36,0.375)$, Fig. 3 shows the total throughput of a multipath CMP-AIMD flow and that of three singlepath AIMD flows that run separately on two paths. As seen, the multipath CMP-AIMD flow approaches the maximum throughput of the single-path flow over the best path, provided that they experience the same packet loss.

Indeed, a multipath transport solution needs to aggregate available bandwidth of multiple paths and offer an improved throughput. However, it is necessary to regulate multipath 


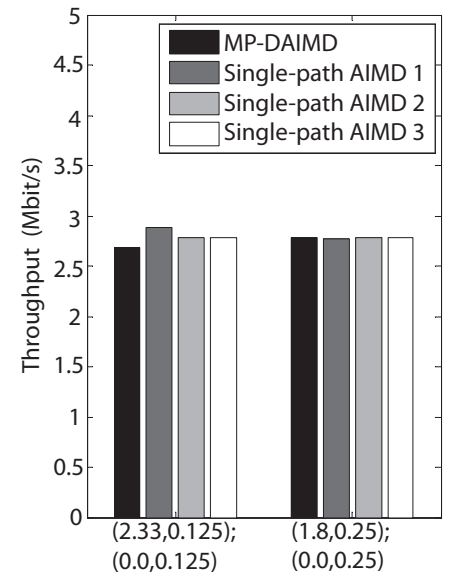

(a) Scenario I.

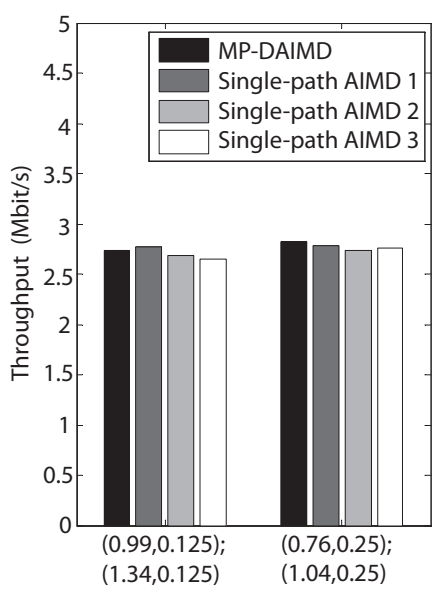

(b) Scenario II.
Fig. 2. Throughput of single-path AIMD and multipath MP-DAIMD flows.

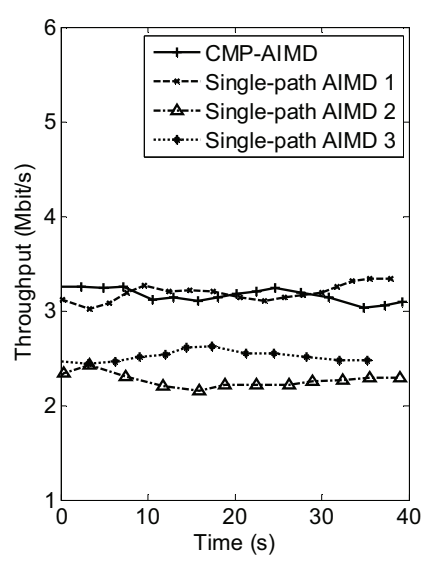

(a) Scenario I.

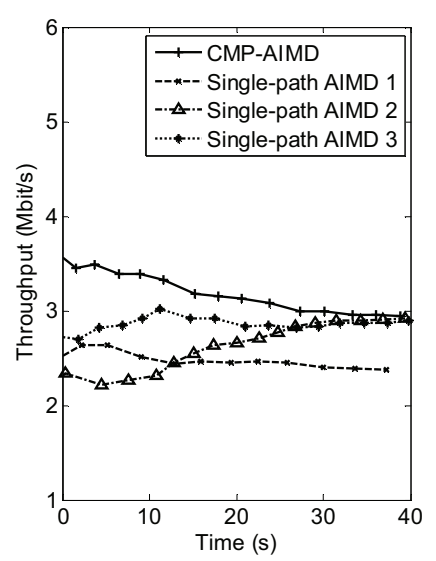

(b) Scenario II.
Fig. 3. Throughput of single-path AIMD and CMP-AIMD multipath flows with $\left(\alpha_{r}, \beta_{r}\right)=(1.36,0.375)$.

flows so that dominant single-path flows are protected from being starved by the aggressive multipath behavior. In Fig. 4, we compare CMP-AIMD to an existing multipath solution MPTCP [4] with respect to the fairness constraint C2 for both the homogeneous and heterogeneous scenarios. Here, three single-path AIMD flows share the bandwidth with a multipath flow, and two pairs of $\left(\alpha_{r}, \beta_{r}\right)$ are considered. As seen, in the presence of single-path flows, CMP-AIMD always satisfies C2 and does not exceed the throughput of single-path flows over the best path. In contrast, the MPTCP-based multipath flow is much more aggressive and taking more bandwidth than single-path flows as MPTCP only addresses fairness with TCP flows.

\section{Conclusions}

In this paper, we focus on the congestion control issue for multipath transmission. To ensure fair bandwidth sharing between multipath and single-path flows, two design constraints are considered in developing congestion control solutions for multipath transmission. On one hand, it is expected from a network operator's perspective that each multipath or singlepath flow obtains an even share of the overall bandwidths if each flow is charged the same. On the other hand, an end
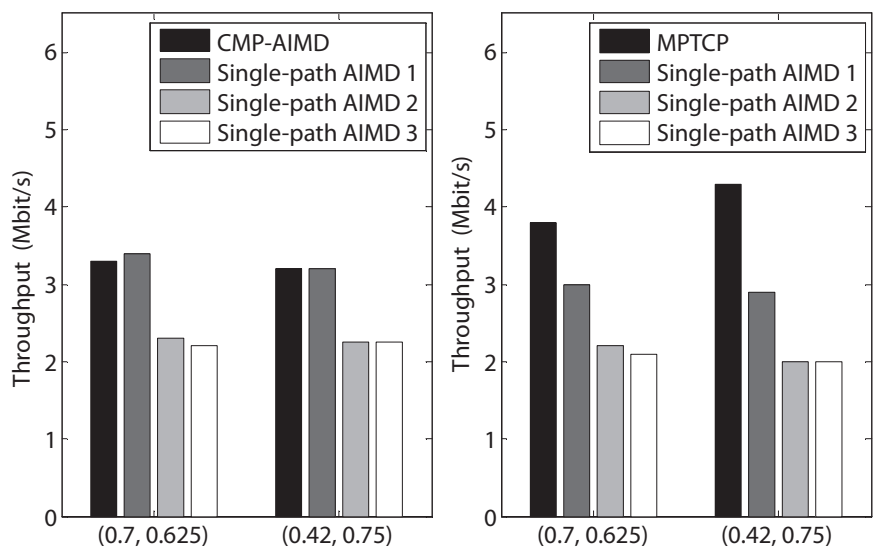

(a) CMP-AIMD in Scenario I.

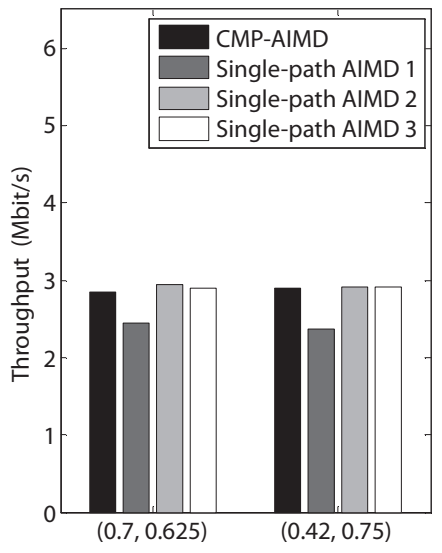

(c) CMP-AIMD in Scenario II. (b) MPTCP in Scenario I.

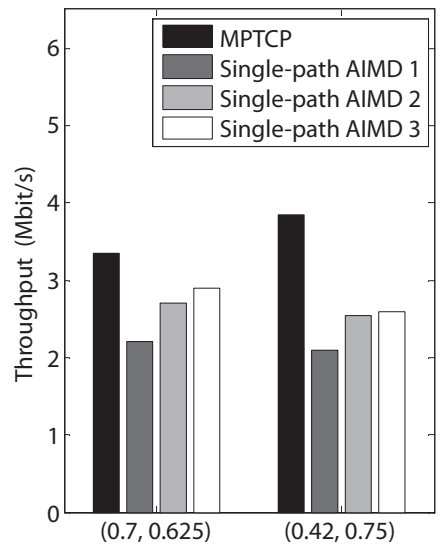

(d) MPTCP in Scenario II.

Fig. 4. Throughput of single-path AIMD and multipath CMP-AIMD and MPTCP flows.

user can be motivated to start multipath transmission if the aggregate throughput of a multipath flow is no less than that of a single-path flow over the best path. Two AIMD-based congestion control solutions, known as $M P$-DAIMD and $C M P$ $A I M D$, are analyzed to achieve the two design goals. We derive the conditions to properly select the AIMD parameters in terms of $(\alpha, \beta)$ pairs and an aggressiveness factor. As shown in the simulation results, the two solutions satisfy the fairness constraints in both homogeneous and heterogeneous scenarios.

\section{REFERENCES}

[1] W. Song and W. Zhuang, "Performance analysis of probabilistic multipath transmission of video streaming traffic over multi-radio wireless devices," IEEE Trans. Wireless Commun., vol. 11, no. 4, pp. 1554-1564, Apr. 2012.

[2] L. Cai, X. Shen, J. Pan, and J. W. Mark, "Performance analysis of TCPfriendly AIMD algorithms for multimedia applications," IEEE Trans. Mutimedia., vol. 7, no. 2, pp. 339-355, Mar. 2005.

[3] A. Ford, C. Raiciu, M. Handley, S. Barre, and J. Iyengar, "Architectural guidelines for multipath TCP development," IETF RFC 6182, Mar. 2011.

[4] C. Raiciu, M. Handly, and D. Wischik, "Coupled congestion control for multipath transport protocols," IETF RFC 6356, Oct. 2011.

[5] D. Wischik, C. Raiciu, A. Greenhalgh, and M. Handley, "Design, implementation and evaluation of congestion control for multipath TCP," in Proc. 2011 USENIX NSDI.

[6] T. A. Le et al., "ecMTCP: an energy-aware congestion control algorithm for multipath TCP," IEEE Commun. Lett., vol. 16, pp. 275-277, 2012.

[7] M. Becke et al., "On the fairness of transport protocols in a multi-path environment," in Proc. 2012 IEEE ICC, pp. 2699-2705.

[8] J. Liu and S. Singh, "ATCP: TCP for mobile ad hoc networks," IEEE J. Sel. Areas Commun., vol. 19, no. 7, pp. 1300-1315, Jul. 2001. 